\title{
Simulation and performance analysis of a novel high-accuracy sheathless microfluidic impedance cytometer with coplanar electrode layout
}

\author{
Federica Caselli*, Paolo Bisegna
}

Department of Civil Engineering and Computer Science, University of Rome Tor Vergata, 00133 Rome, Italy

\begin{abstract}
1 The performance of a novel microfluidic impedance cytometer (MIC) with 2 coplanar configuration is investigated in-silico. The main feature of the de3 vice is the ability to provide accurate particle-sizing despite the well-known 4 measurement sensitivity to particle trajectory. The working principle of the 5 device is presented and validated by means of an original virtual laboratory 6 providing close-to-experimental synthetic data streams. It is shown that a 7 metric correlating with particle trajectory can be extracted from the signal 8 traces and used to compensate the trajectory-induced error in the estimated 9 particle size, thus reaching high-accuracy. An analysis of relevant parameters 10 of the experimental setup is also presented.
\end{abstract}

Keywords: microfluidic impedance cytometry, coplanar electrodes, particle sizing, modeling and simulation

\footnotetext{
*Corresponding author

Email address: caselli@ing.uniroma2.it (Federica Caselli)
} 


\section{Introduction}

In medicine, life science and quality control there is a pressing need to develop simple yet accurate tools for single-cell analysis, which is the new frontier in omics [1]. Electrical phenotyping offers a non-invasive method for the analysis and characterization of particles and cells on the basis of dielectric properties [2]. Besides conventional techniques like dielectrophoresis and electrorotation (e.g., $[3,4,5,6]$ ), the advent of microfluidic technology enabled the development of high-throughput microfluidic impedance cytometers (MICs). Typically, the core of a MIC is a microfluidic chip consisting of a microchannel equipped with microelectrodes and filled with a conductive buffer. An AC voltage is applied to a pair of electrodes, which causes an electric current to flow between them. Upon passage of a cell between the electrodes a current change is measured, providing information on cell size, membrane and intracellular space, according to the frequency of the stimulation voltage [7]. This technology has applications in basic research, diagnostics, or non-invasively probing cell function at the single-cell level (see e.g. the reviews $[8,9]$ and the references therein).

Two main chip designs have been considered in the literature [2]: either chips with electrodes embedded on one side of the channel (coplanar electrodes), or chips with electrodes embedded in facing sides (parallel electrodes). Vertical 3D-electrodes have also been proposed (e.g., [10, 11]). Chips with coplanar configuration are especially attractive, because coplanar electrodes can be easily patterned yielding miniaturized, reproducible, and ultimately low-cost devices $[12,13,14,15,16]$. However, their accuracy is challenged by the dependence of the measured signal on particle trajectory 
within the interrogation volume $[17,18]$, that manifests itself as an error in the estimated particle size ("electrical" diameter), unless any kind of focusing system is used.

The aim of this work is to analyze in-silico a new, easy-to-realize MIC able to provide high-accuracy size estimation without the need for focusing [19]. To this end, synthetic data streams closely mimicking experimental data streams have been generated and processed, by means of an original and versatile virtual laboratory.

The device under evaluation uses a chip with coplanar electrodes, and its operation mode is conceived such that a peculiar electric field distribution is generated within the sensing region. As a consequence, the signal trace recorded upon the passage of a particle exhibits a characteristic shape, whence a new metric can be extracted correlating with particle trajectory height. It is proved in simulation that this metric can be used to compensate for the spurious spread in the measured electrical diameter associated with trajectory height, thus achieving high accuracy.

The paper is organized as follows. In Section 2 the novel MIC is described and the new metric is introduced. Its relationship with particle trajectory height and electrical diameter is investigated in Section 3 by means of a finite element simulation campaign. As a result, a simple strategy to correct the electrical diameter is derived. A parametric analysis with respect to the relevant parameters of the experimental setup is also presented. Finally, a virtual particle-sizing experiment involving dielectric spherical beads with diameter of 5, 6 and $7 \mu \mathrm{m}$ is carried on in Section 4 under different noise levels, showing the effectiveness and soundness of the proposed methodology. 
For the sake of completeness, finite element model equations are reported in Appendix A. Dimensionless equations, elucidating the role of model parameters, are also provided. The relationship among particle velocity, electrical diameter, and particle trajectory within the channel is investigated in Appendix B.

\section{Coplanar electrode high-accuracy microfluidic impedance chip}

A schematic representation of the microfluidic chip considered in this work is depicted in Figure 1(a). It consists of a microfluidic channel $(40 \mu \mathrm{m}$ wide, $21.5 \mu \mathrm{m}$ height), with five electrodes deposited on its floor (30 $\mu \mathrm{m}$ electrode width, $10 \mu \mathrm{m}$ spacing). A Cartesian reference frame is introduced, with the $x, y$ and $z$-axis parallel to the channel width, height and longitudinal axis, respectively. The device is operated as follows (Figure 1(b)): a conductive buffer fills the channel, an AC voltage signal is applied to the central electrode, and the difference in electric current flowing through the lateral electrodes is measured, $I_{\text {Diff. Intermediate electrodes are left floating. }}$. This wiring results in a non-homogeneous electric field distribution along the channel axis (z-direction), characterized by four regions of high field intensity and weak-field regions in between (Figure 1(b)).

When no particle is present in the sensing region, the differential current $I_{\text {Diff }}$ ideally vanishes by symmetry. Upon the passage of a particle, the induced electric field perturbation produces a variation of $I_{\text {Diff. Figure } 1(\mathrm{c})}$ shows the traces (real part) obtained in simulation when a dielectric bead with diameter of $5 \mu \mathrm{m}$ (curve 1), $6 \mu \mathrm{m}$ (curve 2), or $7 \mu \mathrm{m}$ (curve 3) travels through the middle of the channel (see Appendix A for the details of the nu- 

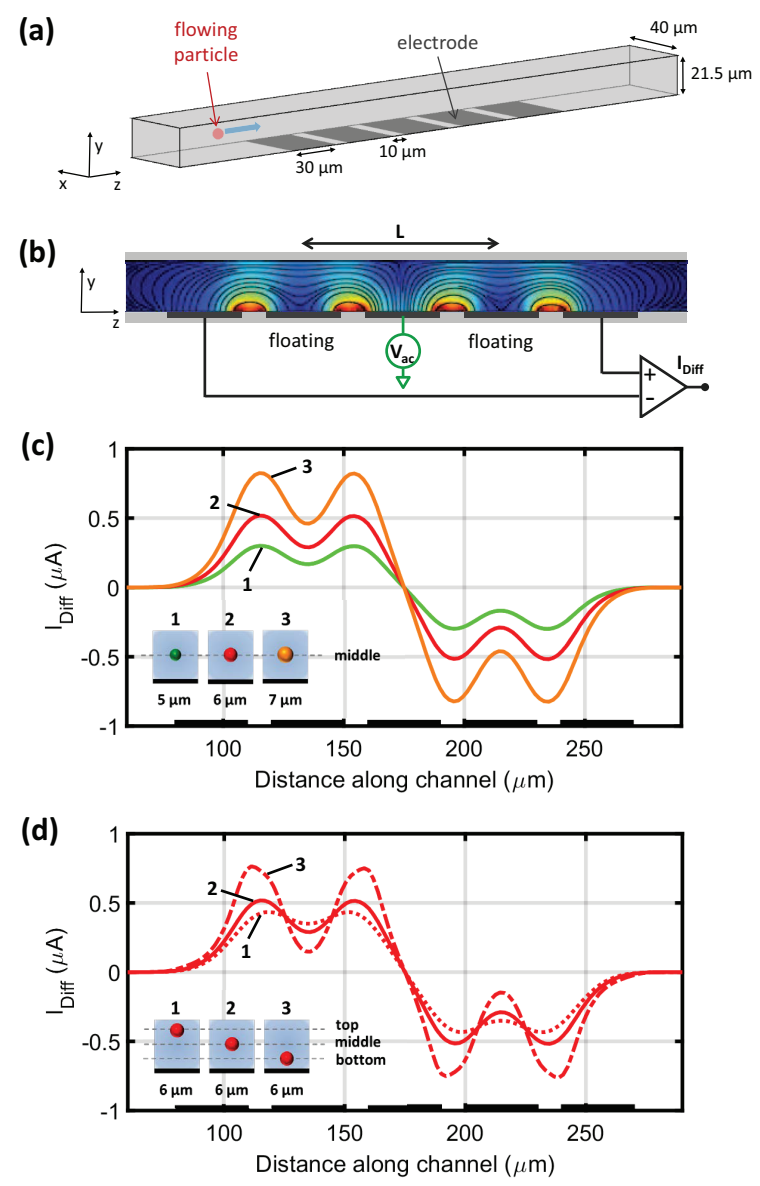

Figure 1: Original coplanar five-electrode MIC. (a) Schematic representation of the microfluidic chip. (b) Operation mode: AC excitation signals are applied to the central electrode, and the difference in current flowing through the lateral electrodes is measured using a differential amplifier; intermediate electrodes are floating. Current lines and electric field magnitude distribution are pictured. (c) Differential signals (real part) recorded when a dielectric bead with diameter of $5 \mu \mathrm{m}$ (curve 1), $6 \mu \mathrm{m}$ (curve 2), or $7 \mu \mathrm{m}$ (curve 3) travels through the middle of the channel. (d) Differential signals (real part) recorded when a dielectric bead with diameter of $6 \mu \mathrm{m}$ travels through the sensing region at three different heights: close to the top of the channel (curve 1), through the middle of the channel (curve 2) or close to the electrodes (curve 3). 


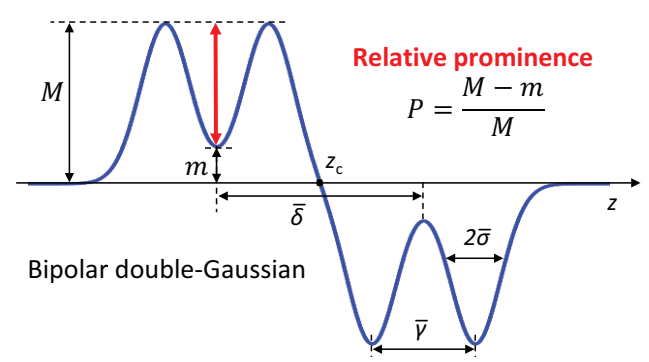

Figure 2: Bipolar double-Gaussian template used as event fitting function. The definition of relative prominence $P$ is also shown.

${ }_{93} \bar{\gamma}, a$ respectively represent control parameters for peak width, peak distance

94 in each double Gaussian, peak amplitude.

95 Peak amplitude is proportional to particle volume [8], hence the cube root

merical model). A bipolar double-Gaussian profile is observed, whose peaks correspond to higher-field regions along the $z$-direction. This profile is well captured by the following template (Figure 2):

$$
\bar{s}(z)=a\left[\bar{g}\left(z-z_{c}+\bar{\delta} / 2\right)-\bar{g}\left(z-z_{c}-\bar{\delta} / 2\right]\right.
$$

with:

$$
\bar{g}(z)=e^{-(z-\bar{\gamma} / 2)^{2} /\left(2 \bar{\sigma}^{2}\right)}+e^{-(z+\bar{\gamma} / 2)^{2} /\left(2 \bar{\sigma}^{2}\right)} .
$$

Here, $z_{c}$ is the $z$-coordinate of the center of the sensing region, $z_{c}=175 \mu \mathrm{m}$; $\bar{\delta} \approx L$, where $L=80 \mu \mathrm{m}$ is twice the electrode pitch (Figure 1(b)); and $\bar{\sigma}$, of $a$ can be used to estimate particle diameter:

$$
D=G a^{1 / 3}
$$$$
97
$$

where $G$ is a proportionality factor depending on device geometric and dielectric properties. Accordingly, $D$ is referred to as electrical diameter. 
However, the electric field intensity decreases away from electrodes in the height direction (Figure 1(b)). As a consequence, peak amplitude also depends on particle trajectory height, i.e. $y$-coordinate. Figure 1(d) shows the simulated traces relevant to a dielectric bead with diameter of $6 \mu \mathrm{m}$ traveling near the top of the channel (curve 1), through the middle of the channel (curve 2), or close to the electrodes (curve 3). Comparing these simulation results with those in Figure 1(c) it is evident that, by looking only at peak amplitude, a $6 \mu \mathrm{m}$ diameter bead flowing close to the electrodes [respectively, near the top of the channel] is hardly distinguishable from a $7 \mu \mathrm{m}$ [respectively, $5 \mu \mathrm{m}$ ] diameter bead passing through the middle of the channel.

On the other hand, the richness of the information contained in the measured signals can be exploited to decouple the effect of particle size and particle trajectory height. As shown by the simulated traces in Figure 1(d), the prominence of the two peaks of the double-Gaussian profile with respect to the saddle in between is higher for particles traveling close to the electrodes (curve 3) than for particles traveling away from the electrodes (curve 1). Because the signal amplitude also depends on particle size, the following normalized metric, referred to as relative prominence, is introduced (Figure 2):

$$
P=\frac{M-m}{M},
$$

where $m$ and $M$ essentially correspond to signal amplitude at saddle and 
peaks, respectively, i.e.:

$$
\begin{aligned}
m=\bar{s}\left(z_{c}-\bar{\delta} / 2\right), \quad M=\left(M_{\text {left }}+M_{\text {right }}\right) / 2 \\
M_{\text {left }}=\bar{s}\left(z_{c}-\bar{\delta} / 2-\bar{\gamma} / 2\right), \quad M_{\text {right }}=\bar{s}\left(z_{c}-\bar{\delta} / 2+\bar{\gamma} / 2\right) .
\end{aligned}
$$

Simple calculus yields the following approximate expression of the relative prominence:

$$
P=1-2 e^{-\bar{\gamma}^{2} /\left(8 \bar{\sigma}^{2}\right)} .
$$

As demonstrated in Section 3, the relative prominence correlates with the height of the particle trajectory: the higher the former, the lower the latter. This metric can therefore be used to correct the electrical particle diameter by means of a simple compensation formula, thus yielding high accuracy in size estimation.

\section{In silico proof of principle}

In order to elucidate the relationship among electrical diameter $D$, particle trajectory height $y$, and relative prominence $P$, a numerical campaign was performed. Dielectric spherical beads with diameter of $6 \mu \mathrm{m}$ were considered. Dielectric beads mimic cell behaviour at frequencies below the MaxwellWagner relaxation (" $\beta$-relaxation", 1-100 MHz) arising from the polarization of the cell membranes [20]. Thirteen equally spaced trajectory heights were simulated, allowing a $1.5 \mu \mathrm{m}$ gap from the microchannel top and bottom walls. Particles were centered along the $x$-axis. ${ }^{1}$ Parameter values adopted

\footnotetext{
${ }^{1}$ Particle trajectories differing only for their $x$-coordinate provide nearly identical signals as a function of the $z$-coordinate, because the electric field is homogeneous along the $x$-axis.
} 
(a)

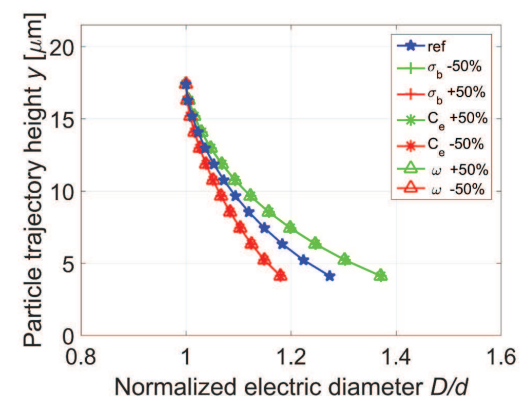

(c)

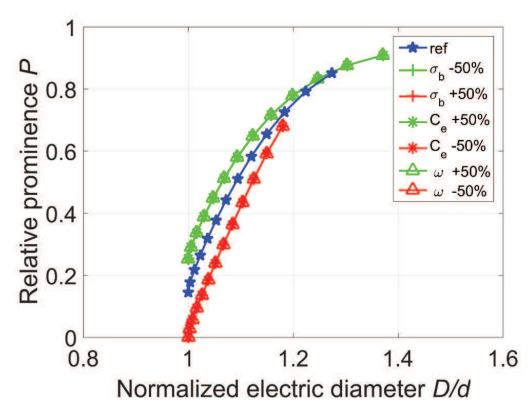

(b)

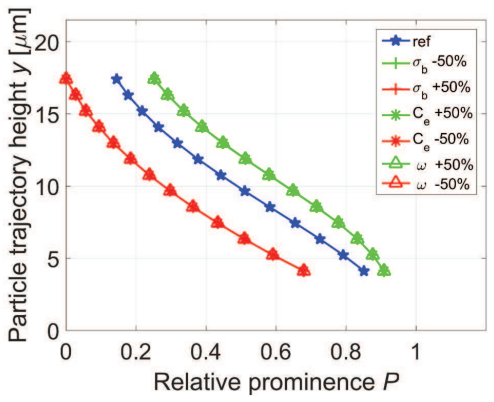

(d)

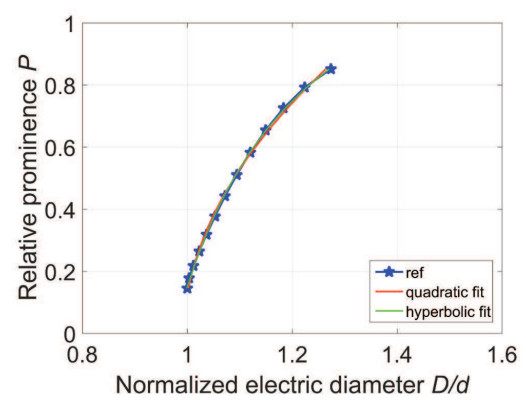

Figure 3: Simulation results relevant to a $6 \mu \mathrm{m}$ diameter bead traveling through trajectories with thirteen different heights. Reference model parameter values (blue pentagrams) as well as $\pm 50 \%$ variations (refer to legend) are considered. (a) Particle trajectory height $y$ vs electrical diameter $D$ normalized by nominal bead diameter $d$. (b) Particle trajectory height $y$ vs relative prominence $P$. (c) Relative prominence $P$ vs normalized electrical diameter $D / d$, (d) fitted with quadratic or hyperbolic model equations (reference model parameter values). 
in the finite-element simulations are reported in Appendix A. Variations of $\pm 50 \%$ with respect to the reference values were also considered for the conductivity of the fluid buffer $\sigma_{\mathrm{b}}$, the electrode double-layer capacitance $C_{\mathrm{e}}$, and the stimulation (circular) frequency $\omega$.

Figure 3(a) shows particle trajectory height $y$ versus electrical diameter $D$ normalized by particle diameter $d$. Considering e.g. the reference parameter set (blue pentagrams), it turns out that $D / d$ varies of about $30 \%$ depending on $y$, thus revealing the positional dependence issue. However, a strong correlation between particle trajectory height $y$ and relative prominence $P$ is observed in Figure 3(b), suggesting that the latter can be a suitable metric to estimate the former. By combining the curves in Figure 3(a) and (b), a relationship between relative prominence $P$ and normalized electrical diameter $D / d$ is obtained (Figure 3(c)). This relationship can be conveniently described, e.g., by a quadratic or hyperbolic function (Figure 3(d)), with model equation:

$$
D / d=c_{1}+c_{2} P+c_{3} P^{2},
$$

or

$$
D / d=\tilde{c}_{1}+\tilde{c}_{2} /\left(P-\tilde{c}_{3}\right)
$$

where $c_{1}, c_{2}, c_{3}$ (or $\tilde{c}_{1}, \tilde{c}_{2}, \tilde{c}_{3}$ ) are fitting parameters. Accordingly, an accurate estimate of the particle diameter $d$ can be derived by respectively correcting the electrical diameter $D$ as follows:

$$
D \text {-corr }=\frac{D}{c_{1}+c_{2} P+c_{3} P^{2}},
$$

or

$$
D \text {-corr }=\frac{D}{\tilde{c}_{1}+\tilde{c}_{2} /\left(P-\tilde{c}_{3}\right)} .
$$


The values of the fitting parameters depend on the experimental setup, and can be obtained experimentally by means of calibration with particles of known size. As a matter of fact, the relationship among electrical diameter $D$, particle trajectory height $y$, and relative prominence $P$ is mainly influenced by the following dimensionless parameter (see Appendix A):

$$
\alpha=\frac{\omega C_{\mathrm{e}} l}{\sigma_{\mathrm{b}}},
$$

where $l$ is a characteristic length of the chip. As shown in Figure 3(a), the spread of the electrical diameter $D$ associated with trajectory height becomes more severe as a consequence of an increase in $\alpha$, that in turn may depend on a decrease in $\sigma_{\mathrm{b}}$ (green plus), or an increase in $C_{\mathrm{e}}$ (green stars) or $\omega$ (green triangles). On the other hand, the spread of the electrical diameter is mitigated by the opposite parameter variations (red curves). Moreover, Figure 3(b) shows that the relative prominence $P$ increases with $\alpha$ for any fixed trajectory height $y$. This trend is reflected in the location and shape of the curve relating the relative prominence $P$ and the normalized electrical diameter $D / d$ (Figure 3(c)). That curve turns out to be best fitted by the quadratic [respectively, hyperbolic] model equation (7) [respectively, (8)] for lower [respectively, higher] values of $\alpha$, thus implying the compensation procedure in equation (9) [respectively, (10)].

\section{A virtual case study: size estimation of 5,6 and $7 \mu \mathrm{m}$ diameter} beads.

In order to test the performance of the proposed compensation procedure, the size estimation of 5,6 and $7 \mu \mathrm{m}$ diameter beads has been addressed in a 
Table 1: Parameter values used in the generation of the synthetic data stream $\mathcal{S}_{\text {mix }}$

\begin{tabular}{lllllllll}
$N_{\mathrm{p}}$ & $d_{1-3}[\mu \mathrm{m}]$ & $\mathrm{CV}_{1-3}[\%]$ & $\rho_{1-3}[\# / \mu \mathrm{l}]$ & $\phi[\mu \mathrm{l} / \mathrm{min}]$ & $n$ & $B W[\mathrm{kHz}]$ & $f_{s}[\mathrm{ksps}]$ & $\sigma_{\mathrm{N}}[\mathrm{nA}]$ \\
\hline 3 & $5,6,7$ & $2.5,1,1$ & $10^{3} / 3$ & 10 & 4 & 20 & 115 & 130 \\
\hline
\end{tabular}

virtual laboratory. To this aim, a synthetic data stream has been generated and subsequently processed, as described in the following.

\subsection{Synthetic data stream generation}

The data stream, denoted by $\mathcal{S}_{\text {mix }}$, is relevant to a mixture of $N_{\mathrm{p}}$ populations of dielectric spherical beads suspended in a conductive buffer at respective concentrations $\rho_{1}, \ldots, \rho_{N_{\mathrm{p}}}$, pumped through the device at a flow rate $\phi$. Population nominal diameters are $d_{1}, \ldots, d_{N_{\mathrm{p}}}$, with coefficient of variations $\mathrm{CV}_{1}, \ldots, \mathrm{CV}_{N_{\mathrm{p}}}$, respectively.

A number $N_{\mathrm{e}}$ of events (i.e, passage of a particle in the sensing region) has been generated. The typical event $e$ is characterized by the following quantities:

- $p_{e} \in\left\{1, \ldots, N_{\mathrm{p}}\right\}$ : population index, denoting the population the event belongs to, drawn from the finite sample space $\left\{1, \ldots, N_{\mathrm{p}}\right\}$ with the probabilities $\rho_{1} / \rho, \ldots, \rho_{N_{\mathrm{p}}} / \rho$, where $\rho=\sum_{p=1}^{N_{\mathrm{p}}} \rho_{p}$ is the total particle concentration;

- $d_{e}$ : particle diameter, drawn from the Gaussian distribution with mean $d_{p_{e}}$ and standard deviation $\sigma_{p_{e}}=\mathrm{CV}_{p_{e}} d_{p_{e}}$;

- $\left(x_{e}, y_{e}\right):(x, y)$-coordinates of the particle trajectory in the channel cross section, drawn from a uniform distribution in the available cross section 
(a)

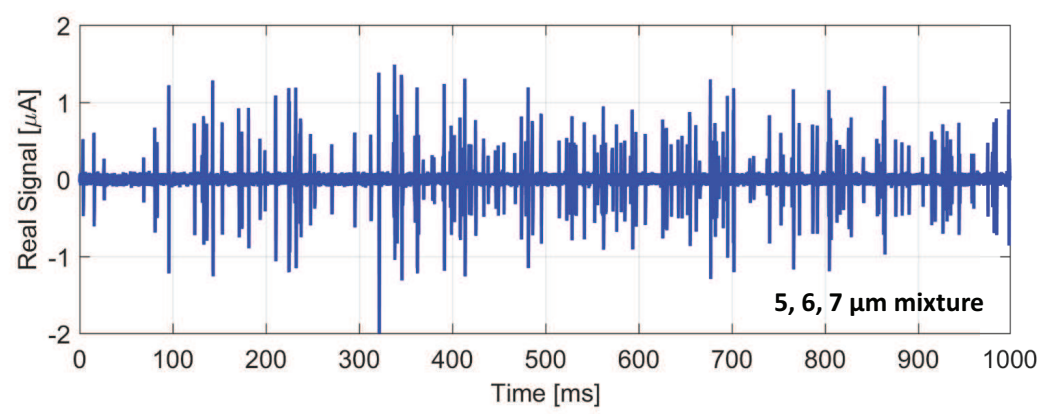

(b)

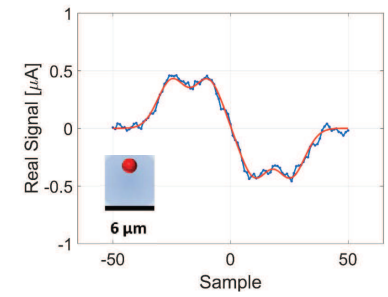

(c)

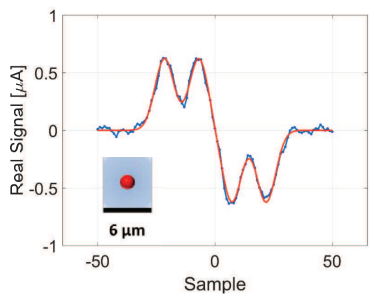

(d)

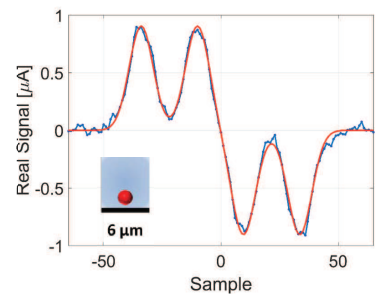

Figure 4: (a) Portion of the synthetic data stream $\mathcal{S}_{\text {mix }}$, relevant to a mixture of 5,6 and $7 \mu \mathrm{m}$ beads. (b)-(d) Exemplary events (blue curves) taken from data stream $\mathcal{S}_{6}$, generated by $6 \mu \mathrm{m}$ diameter beads traveling (b) close to the top of the microchannel, (c) through the middle of the microchannel, and (d) close to the electrodes. Fitting templates are also shown (red curves). 
region (a $1.5 \mu \mathrm{m}$ gap between particle boundary and channel walls has been assumed) $;^{2}$

- $v_{e}$ : particle velocity, determined as a function of $\left(x_{e}, y_{e}\right)$ assuming laminar flow [22] (in fact, Reynolds number is typically in the order of units);

- $t_{e}$ : particle entrance time (i.e., time instant the particle center passes through the entrance cross-section). Occurrence of particles was assumed to be a Poisson process [23]. Accordingly, particle inter-arrival times $\Delta t_{e}$ were drawn from an exponential distribution with rate parameter $\lambda=\phi \rho$.

From the experimental point of view, the signal trace $S(t)$, measuring the differential current $I_{\text {Diff, }}$ is recorded as function of time $t$. Excluding situations of very high particle concentration, particles do not electrically interact with each other. Accordingly, $S(t)$ can be obtained by adding the contributions of the events with entrance time $t_{e} \leq t$ :

$$
S(t)=\sum_{\left\{e: t \geq t_{e}\right\}} S_{p_{e}}\left(x_{e}, y_{e}, z_{e}(t)\right)\left(\frac{d_{e}}{d_{p_{e}}}\right)^{3} .
$$

Because particles essentially experience uniform linear motion in the microchannel, at least over distances of the oder of the sensing region length, the law of motion $z_{e}(t)=v_{e}\left(t-t_{e}\right)$ can be assumed. The function $S_{p_{e}}\left(x_{e}, y_{e}, z_{e}(t)\right)$

\footnotetext{
${ }^{2}$ Some amount of hydrodynamic focusing may be present [21]. However, it is immaterial for the present purpose, so it is neglected here. On the other hand, it could be easily accounted for by introducing an appropriate nonuniform distribution of $\left(x_{e}, y_{e}\right)$ in the channel cross section.
} 
is independent of $x_{e}$, because the electric field is homogeneous along the $x$ axis. Its value at $\left(y_{e}, z_{e}(t)\right)$ is obtained by means of $2 \mathrm{D}$ interpolation of a repository of pre-computed values over a regular grid of $(y, z)$ locations, for each nominal population diameter (see e.g., Figure 1(c)-(d)). Finally, the factor $\left(d_{e} / d_{p_{e}}\right)^{3}$ in equation (12) accounts for the actual particle diameter, which is normally distributed around the nominal population diameter.

Additive white noise with standard deviation $\sigma_{\mathrm{N}}$ was added to the data stream. A filter consisting of $n$ first-order filtering steps was implemented, with resulting filter bandwith $B W$. A sampling frequency $f_{s}$ was assumed.

The parameter values used in the generation of the synthetic data stream $\mathcal{S}_{\text {mix }}$, comprising 54000 events, are reported in Table 1 . Those values are typical of experimental settings (e.g., [24]). A $\pm 50 \%$ variation of the noise level was also considered while testing the method (Section 4.3).

Following an analogous procedure, three additional data streams, $\mathcal{S}_{5}, \mathcal{S}_{6}$, and $\mathcal{S}_{7}$, relevant to single populations of dielectric spherical beads with diameter respectively of 5,6 and $7 \mu \mathrm{m}$, were also built (comprising 18000 events each).

Figure 4 shows one second of the syntectic data stream $\mathcal{S}_{\text {mix }}$, along with the zoom of three exemplary events taken from $\mathcal{S}_{6}$.

\subsection{Data stream processing}

The synthetic data streams were processed with an in-house software toolbox. First, event detection in the data stream was performed using the algorithm described in [25]. With the present flow rate and sample concentration, a theoretical throughput of 166 events per second was computed. A throughput of about 130 events per second was obtained, because the 
segmentation algorithm rejects coincidences.

For each detected event, template fitting and feature extraction were carried on as follows. The counterpart in time, $s(t)$, of the bipolar doubleGaussian template $\bar{s}(z)$ introduced in equation (1) was used:

$$
s(t)=a\left[g\left(t-t_{c}+\delta / 2\right)-g\left(t-t_{c}-\delta / 2\right)\right],
$$

with:

$$
g(t)=e^{-(t-\gamma / 2)^{2} /\left(2 \sigma^{2}\right)}+e^{-(t+\gamma / 2)^{2} /\left(2 \sigma^{2}\right)} .
$$

This template depends on five parameters: central time moment, $t_{c}$; transit time, $\delta$; peak width control, $\sigma$; peak distance control, $\gamma$, and peak amplitude control, $a$. Parameters $\delta, \sigma$, and $\gamma$ are related to their space-domain counterparts respectively by $\delta=\bar{\delta} / v_{e}, \sigma=\bar{\sigma} / v_{e}$, and $\gamma=\bar{\gamma} / v_{e}$. The fitting parameters $a, \gamma$, and $\sigma$ were used to compute the electrical diameter $D$ and the relative prominence $P$, respectively from equations (3) and (6). In turn, the corrected electrical diameter $D$-corr was obtained from $D$ and $P$ by means of equation (10) or (9).

The particle velocity $v_{e}$ should be considered unknown from the experimental point of view. However, recalling that $\bar{\delta} \approx L$, it can be estimated by the transit time $\delta[26,24]$ :

$$
v_{e}=\bar{\delta} / \delta \approx L / \delta=V
$$

The estimate $V$ is referred to as "electrical" velocity, and is compared to the "true" velocity $v_{e}$ in Appendix B.

\subsection{Particle-sizing results}

Figure 5(a)-(c) show density plots of the relative prominence $P$ versus the electrical diameter $D$, respectively for 5,6 and $7 \mu \mathrm{m}$ diameter beads 
(a)

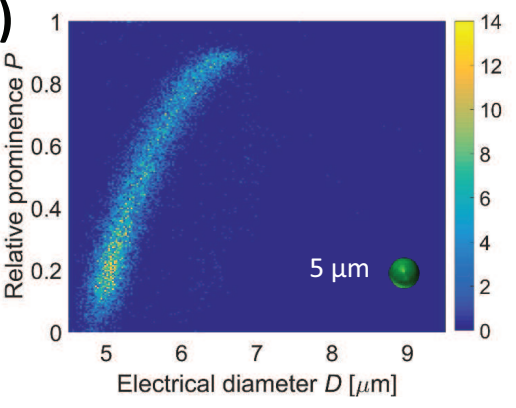

(b)

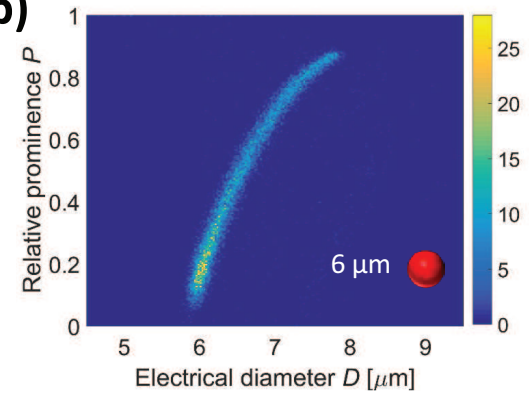

(c)

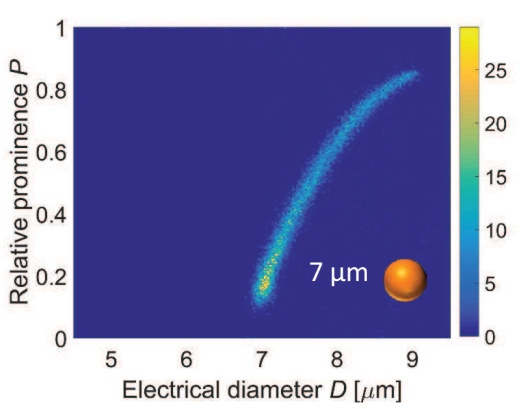

(d)

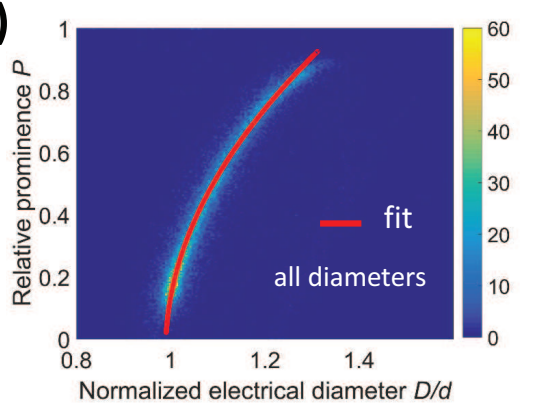

Figure 5: (a)-(c) Density plot of populations of dielectric spherical beads of different sizes, with the relative prominence $P$ plotted against the electrical diameter $D$. (a) $5 \mu \mathrm{m}$ diameter beads $\left(\mathcal{S}_{5}\right)$, (b) $6 \mu \mathrm{m}$ diameter beads $\left(\mathcal{S}_{6}\right)$, (c) $7 \mu \mathrm{m}$ diameter beads $\left(\mathcal{S}_{7}\right)$. (d) Density plot of the relative prominence $P$ against the electrical diameter $D$ normalized by the nominal bead diameter $d$. The density plots relevant to the three individual populations of beads $\left(\mathcal{S}_{5}, \mathcal{S}_{6}\right.$, and $\left.\mathcal{S}_{7}\right)$ are plotted together and overlap. The quadratic fit $D / d=c_{1}+c_{2} P+c_{3} P^{2}$ is shown as red line (fit parameters reported in Table 2, last row). 


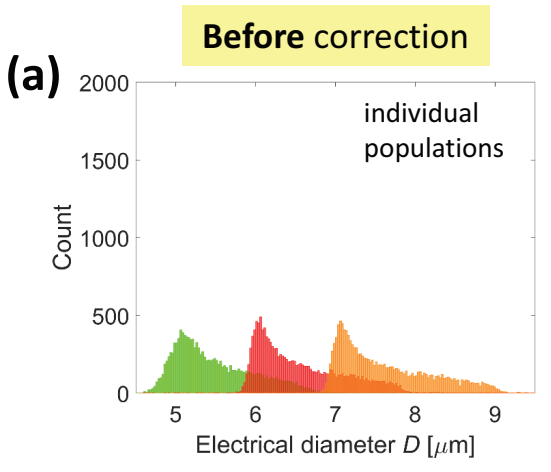

(d)

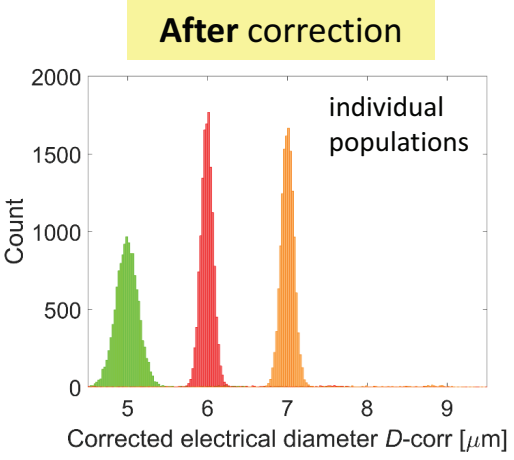

(b)

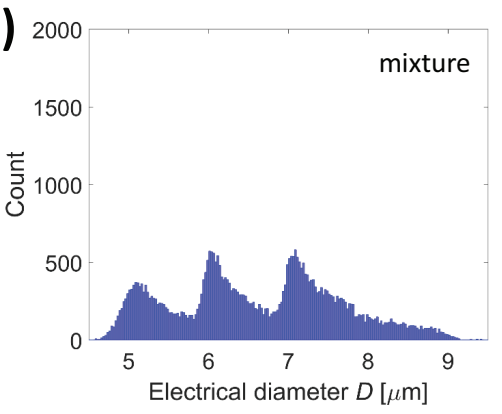

(c)

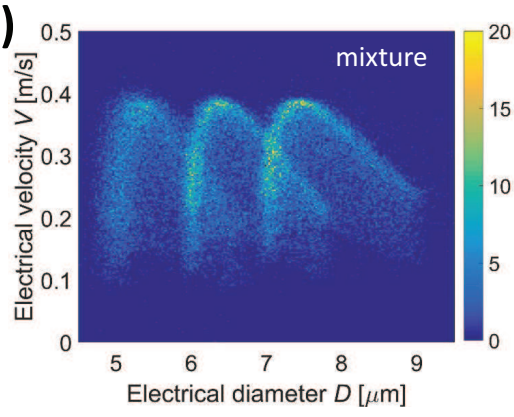

(e)

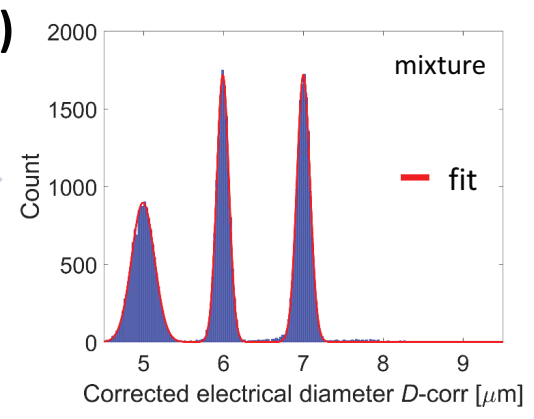

(f)

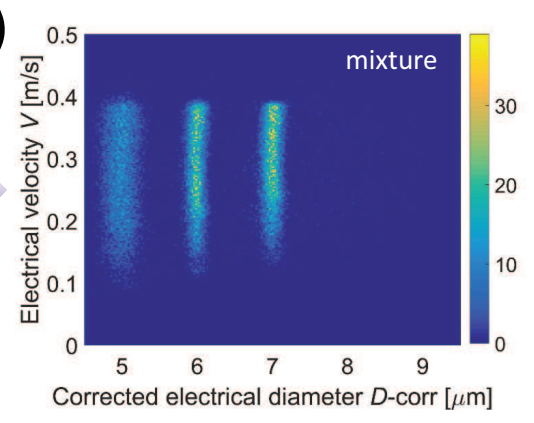

Figure 6: Histogram of the electrical diameter of (a) individual populations of 5, 6 and $7 \mu \mathrm{m}$ diameter beads $\left(\mathcal{S}_{5}, \mathcal{S}_{6}\right.$, and $\left.\mathcal{S}_{7}\right)$ and (b) the mixed sample $\left(\mathcal{S}_{\text {mix }}\right)$, showing significant spread and asymmetry. After compensation (d)-(e), almost perfect Gaussian distributions are found. (c) and (f) show density plots of particle velocity vs electrical diameter for the mixture of beads (c) before and (f) after correction. In (f) each population of beads has the same electrical diameter regardless of velocity and therefore trajectory position through the channel. 
Table 2: Parameters of quadratic model equation $D / d=c_{1}+c_{2} P+c_{3} P^{2}$ used to fit data plotted in Figure 5(d) (individual bead populations or whole ensemble).

\begin{tabular}{llll}
$d[\mu \mathrm{m}]$ & $c_{1}$ & $c_{2}$ & $c_{3}$ \\
\hline 5.0 & 0.99 & 0.026 & 0.35 \\
6.0 & 0.99 & 0.031 & 0.34 \\
7.0 & 0.99 & 0.023 & 0.35 \\
all & 0.99 & 0.028 & 0.35 \\
\hline
\end{tabular}

Table 3: CV of the corrected diameters under different noise level.

\begin{tabular}{lcccc}
$d[\mu \mathrm{m}]$ & CV-theoretical & \multicolumn{3}{c}{$\mathrm{CV}$-estimated } \\
\cline { 3 - 5 } & & \multicolumn{3}{c}{ noise level } \\
& & $-50 \%$ & ref & $+50 \%$ \\
\hline 5.0 & $2.5 \%$ & $2.8 \%$ & $2.9 \%$ & $3.1 \%$ \\
6.0 & $1.0 \%$ & $1.2 \%$ & $1.3 \%$ & $1.5 \%$ \\
7.0 & $1.0 \%$ & $1.1 \%$ & $1.2 \%$ & $1.3 \%$ \\
\hline
\end{tabular}


(i.e., $\mathcal{S}_{5}, \mathcal{S}_{6}$, and $\mathcal{S}_{7}$ ). A common trend is observed for the three populations. Figure 5(d) collects the density plots of $P$ against the electrical diameter $D$ normalized by the nominal diameter $d$, for the three populations of beads (i.e., $\mathcal{S}_{5}, \mathcal{S}_{6}$, and $\mathcal{S}_{7}$, plotted in the same graph). Because the measured signal is proportional to particle volume, these density plots overlap. The data is fitted to the quadratic function introduced in equation (7). For each population, parameter values $c_{1}, c_{2}$, and $c_{3}$ are reported in Table 2 , along with the values obtained by considering all the populations together.

Figure 6(a) and (b) show histograms of the electrical diameter $D$ of (a) individual particle populations (i.e., $\mathcal{S}_{5}, \mathcal{S}_{6}$, and $\mathcal{S}_{7}$ ) and (b) mixed sample $\mathcal{S}_{\text {mix }}$. As expected [14], the distribution has a significant spread and asymmetry, due to the positional dependence issue. The compensation procedure introduced in equation (9) was then implemented, using $c_{1}, c_{2}$ and $c_{3}$ reported in the last row of Table 2. The corrected diameters are plotted in Figure 6(d) and (e) showing an almost perfect Gaussian distribution. Fitting a Gaussian allows the CVs to be calculated as follows (Figure 6(e)): 2.9\%, 1.3\%, and $1.2 \%$, for the 5,6 and $7 \mu \mathrm{m}$ diameter beads respectively. These values are quite close to the theoretical values of $2.5 \%, 1.0 \%$, and $1.0 \%$. The CVs obtained in case of reduced or augmented noise level are reported in Table 3, showing good algorithm performance also with reduced signal-to-noise ratio. The submicron resolution in particle size estimation demonstrated in Figure 6(e) enables accurate size-based particle discrimination, which has significant applications in medicine and life sciences, e.g. to discriminate between cell types, or to investigate cell growth, activation and cell-cycle progression. Figure 6(c) shows density plots of electrical velocity versus electrical di- 
ameter for the mixture of dielectric spherical beads $\left(\mathcal{S}_{\text {mix }}\right)$. An insight on the peculiar shape drawn by the data of each bead population, often reported in the literature, is provided in Appendix B. The corrected data are reported in Figure 6(f), demonstrating that all particles of a given size range have the same corrected electrical diameter irrespective of their velocity, which in turn is related to trajectory through the channel.

\section{Conclusions}

Numerical modeling is a powerful tool for the design and optimization of lab-on-chip devices (e.g, [27, 28, 29, 30]), and has been extensively used in impedance cytometry (e.g., $[17,31,15,32])$. In this work, an original virtual laboratory has been presented, enabling the generation of data streams closely mimicking experimental traces, and easily adaptable to different designs of microfluidic impedance chips.

The virtual laboratory has been exploited to demonstrate the working principle and the performance of a novel microfluidic impedance cytometer enabling high-accuracy size-estimation. The results of the numerical campaigns proved the soundness and robustness of the proposed particle-sizing approach, which has potential applications in high-impact fields like environmental monitoring, food quality control, and point-of-care diagnostics.

\section{Conflict of interest}

None declared. 
Table A.4: Reference parameter values used in the simulations

\begin{tabular}{llllll}
\hline$\omega(\mathrm{rad} / \mathrm{s})$ & $C_{\mathrm{e}}\left(\mathrm{mF} / \mathrm{m}^{2}\right)$ & $\sigma_{\mathrm{b}}(\mathrm{S} / \mathrm{m})$ & $\varepsilon_{\mathrm{b}} / \varepsilon_{\mathrm{v}}$ & $\sigma_{\mathrm{p}}(\mathrm{S} / \mathrm{m})$ & $\varepsilon_{\mathrm{p}} / \varepsilon_{\mathrm{v}}$ \\
\hline $2 \pi \times 10^{6}$ & 33 & 1.1 & 80 & $6.6 \times 10^{-4}$ & 2.5 \\
\hline
\end{tabular}

\section{Funding}

This work was supported by the Scientific Independence of Young Researchers Programme (SIR 2014) under Grant RBSI14TX20-MUSIC "Multidimensional Single-Cell Microfluidic Impedance Cytometry".

\section{Ethical approval}

Not required.

\section{Appendix A. Finite element model equations}

Model equations have been described elsewhere (e.g., [33, 31]), and are summarized here for the sake of completeness. The device is modeled as the union of two homogeneous conducting regions $\Omega_{\mathrm{p}}$ and $\Omega_{\mathrm{b}}$, representing the particle and the fluid buffer, respectively. Their complex conductivities $\sigma_{\mathrm{p}}^{*}$ and $\sigma_{\mathrm{b}}^{*}$ are given by $\sigma_{k}^{*}=\sigma_{k}+\mathrm{i} \omega \varepsilon_{k} \varepsilon_{\mathrm{v}}, k \in\{\mathrm{p}, \mathrm{b}\}$, where $\varepsilon_{\mathrm{v}}$ is the permittivity of free space, and $\sigma_{k}$ and $\varepsilon_{k}$ are the conductivity and relative permittivity of the media, respectively; $\omega$ denotes the circular frequency, and $\mathrm{i}$ is the imaginary unit. Continuity of electric potential and of normal current flux density is enforced at the particle surface $\Gamma$. The boundary of the domain is divided into an insulating part $\left(\partial \Omega_{\mathrm{ne}}\right)$, and a part covered by electrodes $\left(\partial \Omega_{\mathrm{e}}\right)$. 
In the Fourier domain, the electrical problem is stated as follows:

$$
\begin{aligned}
-\operatorname{div}\left(\sigma^{*} \nabla \Psi\right) & =0, & & \text { in } \Omega_{\mathrm{p}} \cup \Omega_{\mathrm{b}} ; \\
\llbracket \sigma^{*} \nabla \Psi \cdot \boldsymbol{n} \rrbracket & =0, & & \text { on } \Gamma ; \\
\llbracket \Psi \rrbracket & =0, & & \text { on } \Gamma,
\end{aligned}
$$

where $\Psi$ is the electric potential phasor, $\sigma^{*}=\sigma_{k}^{*}$ in $\Omega_{k}, k \in\{\mathrm{p}, \mathrm{b}\}$, div and $\nabla$ respectively denote the divergence and gradient operators, $\llbracket \cdot \rrbracket$ is the jump of the enclosed quantity across $\Gamma$, and $\boldsymbol{n}$ denotes the outer unit normal vector. An insulating boundary condition is applied on the boundaries not covered by electrodes

$$
\sigma_{\mathrm{b}}^{*} \nabla \Psi \cdot \boldsymbol{n}=0, \quad \text { on } \partial \Omega_{\mathrm{ne}} .
$$

On the $i$-th electrode $\left(\partial \Omega_{\mathrm{e}_{i}}\right)$, the following electrode equation holds

$$
Y_{\mathrm{e}}\left(\Psi_{i}-\Psi\right)=\sigma_{\mathrm{b}}^{*} \nabla \Psi \cdot \boldsymbol{n}, \quad \text { on } \partial \Omega_{\mathrm{e}_{i}},
$$

where $Y_{\mathrm{e}}=G_{\mathrm{e}}+\mathrm{i} \omega C_{\mathrm{e}}$ is the double-layer admittance per unit area, expressed in terms of conductance $G_{\mathrm{e}}$ and capacitance $C_{\mathrm{e}}$ per unit area, and $\Psi_{i}$ is the electrode potential. The inward current through electrode $i$ is given by

$$
I_{i}=\int_{\partial \Omega_{\mathrm{e}_{i}}} \sigma_{\mathrm{b}}^{*} \nabla \Psi \cdot \boldsymbol{n} \mathrm{d} A, \quad \text { on } \partial \Omega_{\mathrm{e}_{i}} .
$$

For a floating electrode, the relevant potential $\Psi_{i}$ is unknown and the constraint $I_{i}=0$ is enforced.

Reference parameter values used in the simulations are relevant to the experimental setup described in the companion experimental paper [19] and are reported in Table A.4. An electric potential of $4 \mathrm{~V}$ was applied to the central electrode (Figure 1(a)-(b)). 
Quadratic Lagrangian tetrahedral elements were used to interpolate the electric potential $\Psi$. The typical mesh involved about 100,000 tetrahedral elements and 150,000 degrees of freedom. The computational time required for the computation of the differential current $I_{\text {Diff }}$ for one $z$-position was about $30 \mathrm{~s}$ on an Intel(R) Xeon(R) CPU E5-2660 v3 @ $2.60 \mathrm{GHz}$ processor with 128 GB RAM.

In order to obtain dimensionless counterparts of equations (A.1)-(A.5), a characteristic length $l$ of the chip (e.g., the electrode pitch), and a characteristic potential value $\Psi_{o}$ are introduced. Accordingly, dimensionless Cartesian coordinates $(\bar{x}, \bar{y}, \bar{z})$ and electric potential $\bar{\Psi}$ are defined, respectively given by

$$
\bar{x}=x / l, \quad \bar{y}=y / l, \quad \bar{z}=z / l, \quad \bar{\Psi}=\Psi / \Psi_{o} .
$$

Hence, equations (A.1)-(A.5) are transformed into:

$$
\begin{aligned}
-\operatorname{div}(\nabla \bar{\Psi})=0, & \text { in } \bar{\Omega}_{\mathrm{p}} \cup \bar{\Omega}_{\mathrm{b}} ; \\
\left.\nabla \bar{\Psi} \cdot \boldsymbol{n}\right|_{\mathrm{b}}=\left.\beta^{*} \nabla \bar{\Psi} \cdot \boldsymbol{n}\right|_{\mathrm{p}}, & \text { on } \bar{\Gamma} ; \\
\llbracket \bar{\Psi} \rrbracket=0, & \text { on } \bar{\Gamma}, \\
\nabla \Psi \cdot \boldsymbol{n}=0, & \text { on } \partial \bar{\Omega}_{\mathrm{ne}} ; \\
\alpha^{*}\left(\bar{\Psi}_{i}-\bar{\Psi}\right)=\nabla \bar{\Psi} \cdot \boldsymbol{n}, & \text { on } \partial \bar{\Omega}_{\mathrm{e}_{i}} .
\end{aligned}
$$

Here div and $\nabla$ respectively denote the divergence and gradient operators with respect to $(\bar{x}, \bar{y}, \bar{z})$, and are computed on the scaled domains (denoted with an overbar). Moreover, the following dimensionless parameters are introduced:

$$
\alpha^{*}=\frac{l Y_{\mathrm{e}}}{\sigma_{\mathrm{b}}^{*}}, \quad \beta^{*}=\frac{\sigma_{\mathrm{p}}^{*}}{\sigma_{\mathrm{b}}^{*}} .
$$


In the radio-frequency range, $G_{\mathrm{e}}$ is negligible with respect to $\omega C_{\mathrm{e}}$, and $\omega \varepsilon_{\mathrm{b}} \varepsilon_{\mathrm{v}}$ is negligible with respect to $\sigma_{\mathrm{b}}$ for a conductive buffer. Moreover, for a dielectric bead, $\left|\sigma_{\mathrm{p}}^{*}\right| \ll\left|\sigma_{\mathrm{b}}^{*}\right|$, so that:

$$
\alpha^{*} \approx \mathrm{i} \alpha, \quad\left|\beta^{*}\right| \ll 1 .
$$

with $\alpha$ given in equation (11). For the parameter values reported in Table A.4, assuming $l=40 \mu \mathrm{m}$, it turns out that $\alpha=7.5, \alpha^{*}=0.03+\mathrm{i} 7.5$, $\left|\beta^{*}\right|=6.1 \times 10^{-4}$.

As noted in Section 3, the solution of Problem (A.1)-(A.5) for a given chip geometry (hence, the relationship among electrical diameter $D$, particle trajectory height $y$, and relative prominence $P$ ) is mainly influenced by the dimensionless parameter $\alpha$.

\section{Appendix B. Mapping between $(x, y)$-plane and $(D, V)$-plane.}

The density plots of electrical velocity $V$ versus electrical diameter $D$ for the mixture of dielectric spherical beads $\left(\mathcal{S}_{\text {mix }}\right)$ reported in Figure 6(c) exhibit peculiar curved shapes (one for each bead population). Similar shapes have been reported in the literature (e.g., $[34,14]$ ). They depend on the combined effects of velocity distribution inside the channel and positional dependence of electrical diameter.

In order to gain insight into this feature, a noise-free data stream relevant to a single population of $6 \mu \mathrm{m}$ beads with identical diameter (vanishing $\mathrm{CV}$ ) was generated. It was used to construct the $2 \mathrm{D}$ mapping which associates a point $(D, V)$ to every bead center location $(x, y)$ in the channel cross section (Figure B.7). The image of this mapping is the region $\mathcal{R}$ in the $(D, V)$-plane 
(a)

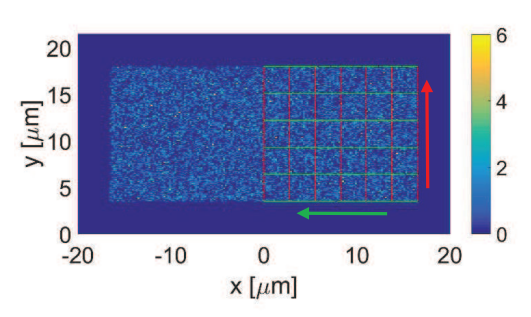

(b)

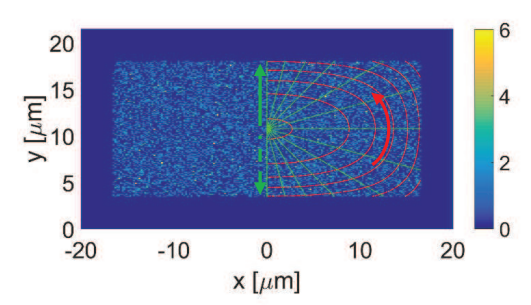

(c)

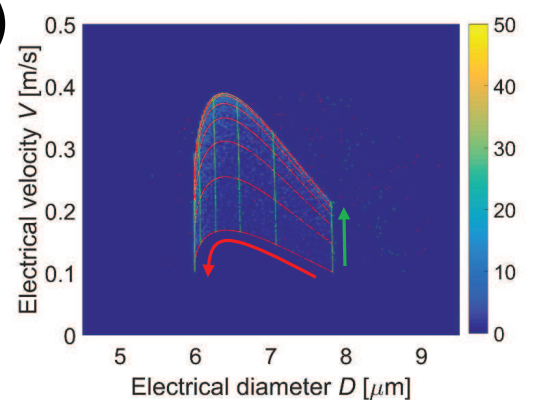

(d)

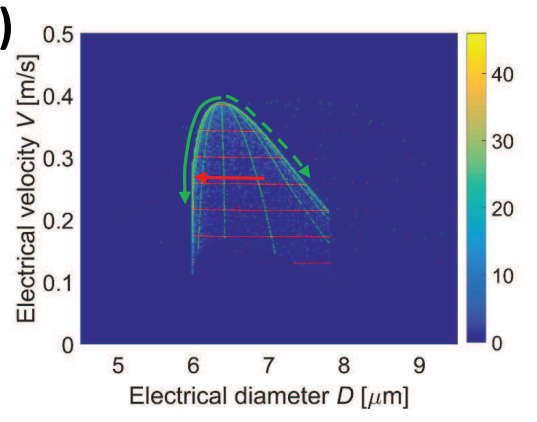

Figure B.7: (a)-(b) Density plot of $x$ - and $y$-coordinates of event centers (uniformly distributed in the channel cross-section, allowing a $1.5 \mu \mathrm{m}$ gap of the $6 \mu \mathrm{m}$ diameter beads from the microchannel walls). Additional events along (a) iso- $x$ and iso- $y$ lines, or (b) iso- $v$ and iso- $\theta$ lines, are marked in red and green, respectively. (c)-(d) Density plots of electrical velocity $V$ vs electrical diameter $D$ relevant to particle distributions in (a) and (b), respectively.

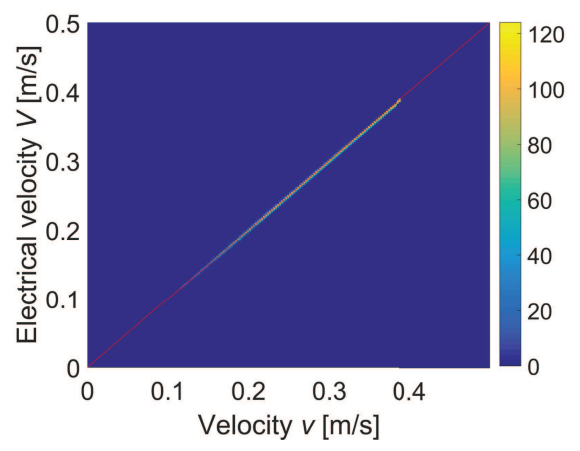

Figure B.8: Density plot of electrical velocity $V$ plotted against the actual velocity $v$. 
(Figure B.7(c),(d)), whose boundary is composed by a top and a bottom curved contour, and by a left and a right straight line.

Besides events uniformly distributed in the channel cross-section (Section 4.1), auxiliary events were generated, relevant to bead centers with $(x, y)$-coordinates distributed along two suitable grids: (i) a Cartesian grid with iso- $x$ and iso- $y$ lines (Figure B.7(a)), and (ii) a grid comprising iso- $v$ and iso- $\theta$ lines (Figure B.7(b)), where $\theta$ denotes the polar angle. The iso- $v$ lines have an approximately elliptic shape, according to the velocity distribution in steady state, hydrodynamically fully developed, laminar flow for Newtonian fluids in rectangular channels [22]. Only one half of the channel in Figure B.7(a),(b) is covered by grids, due to symmetry with respect the $y$ axis.

The analysis of Figure B.7 reveals that:

- iso-y lines (Figure B.7(a)) are mapped onto iso-D lines (Figure B.7(c)). In fact, the electric field is homogeneous along the $x$-axis, so that different $x$ values yield the same value of $D$; on the other hand, the quotient map $y \rightarrow D$ just defines the positional dependence issue addressed in this paper (the higher $y$, the lower $D$ ). In particular, the bottom [respectively, the top] iso- $y$ line is mapped on the right [respectively, left] straight line of the boundary of $\mathcal{R}$;

- iso- $v$ lines (Figure B.7(b)) are mapped onto iso- $V$ lines (Figure B.7(d)), proving that the processing algorithm described in Section 4.2 returns the correct velocity value. This is further emphasized by the density plot of the electrical velocity $V$ versus the actual velocity $v$ reported in Figure B.8, showing excellent correlation along the bisector line $V=v$; 
- iso- $x$ lines (Figure B.7(a)) are mapped onto curved contours (Figure B.7(c)). The closer to the center is the iso- $x$ line, the higher is the curved contour. In particular, the central [respectively, the lateral] iso- $x$ line is mapped on the top [respectively, bottom] curved boundary of $\mathcal{R}$. Moreover, curved contours, images of equispaced iso- $x$ lines, accumulate in the upper part of $\mathcal{R}$, thus emphasizing the top curved profile of $\mathcal{R}$ even when particle centers are uniformly distributed in the channel cross section;

- the right [respectively, left] branch of the top curved contour of $\mathcal{R}$ (Figure B.7 $(\mathrm{d}))$ is the image of the $\theta=-\pi / 2$ [respectively, $\theta=+\pi / 2$ ] isoline (Figure B.7(c)), to which intermediate equispaced $\theta$-isolines tend to accumulate. The top vertex of $\mathcal{R}$ is the image of the channel center.

The insight gained by this analysis could be very helpful in interpreting experimental results involving, e.g., passive or active particle focusing mechanisms.

\section{References}

[1] D. Wang, S. Bodovitz, Single cell analysis: the new frontier in omics, Trends in Biotechnology 28 (6) (2010) 281-290. doi:j.tibtech.2010.03.002.

[2] K. C. Cheung, M. Di Berardino, G. Schade-Kampmann, M. Hebeisen, A. Pierzchalski, J. Bocsi, A. Mittag, A. Tárnok, Microfluidic impedancebased flow cytometry, Cytometry Part A 77 (7) (2010) 648-666. doi:10.1002/cyto.a.20910. 
[3] J. Gimsa, A comprehensive approach to electro-orientation, electrodeformation, dielectrophoresis, and electrorotation of ellipsoidal particles and biological cells, Bioelectrochemistry 54 (1) (2001) 23-31. doi:10.1016/S0302-4598(01)00106-4.

[4] S.-I. Han, Y.-D. Joo, K.-H. Han, An electrorotation technique for measuring the dielectric properties of cells with simultaneous use of negative quadrupolar dielectrophoresis and electrorotation, Analyst 138 (2013) 1529-1537. doi:10.1039/C3AN36261B.

[5] A. Rohani, W. Varhue, Y.-H. Su, N. S. Swami, Electrical tweezer for highly parallelized electrorotation measurements over a wide frequency bandwidth, Electrophoresis 35 (12-13) (2014) 1795-1802. doi:10.1002/elps.201400021.

[6] T. Lannin, W. Su, C. Gruber, I. Cardle, C. Huang, F. Thege, B. Kirby, Automated electrorotation shows electrokinetic separation of pancreatic cancer cells is robust to acquired chemotherapy resistance, serum starvation, and EMT., Biomicrofluidics 10 (5) (2016) 064109. doi:10.1063/1.4964929.

[7] S. Gawad, K. Cheung, U. Seger, A. Bertsch, P. Renaud, Dielectric spectroscopy in a micromachined flow cytometer: theoretical and practical considerations, Lab Chip 4 (3) (2004) 241-251. doi:10.1039/b313761a.

[8] T. Sun, H. Morgan, Single-cell microfluidic impedance cytometry: a review, Microfluid. Nanofluid. 8 (4) (2010) 423-443. doi:10.1007/s10404010-0580-9. 
[9] J. Chen, C. Xue, Y. Zhao, D. Chen, M. Wu, J. Wang, Microfluidic impedance flow cytometry enabling high-throughput single-cell electrical property characterization, Int. J. Mol. Sci. 16 (5) (2015) 9804-9830. doi:10.3390/ijms16059804.

[10] S. Kilchenmann, E. Rollo, E. Bianchi, C. Guiducci, Metal-coated silicon micropillars for freestanding 3D-electrode arrays in microchannel, Sens. Act. B Chem. 185 (2013) 713-719. doi:10.1016/j.snb.2013.05.037.

[11] S. Kilchenmann, E. Rollo, P. Maoddi, C. Guiducci, Metal-coated SU8 structures for high-density 3-D microelectrode arrays, J. Microelectromech. Sys. 25 (3) (2016) 425-431. doi:10.1109/JMEMS.2016.2539000.

[12] J. Hong, D. S. Yoon, S. K. Kim, T. S. Kim, S. Kim, E. Y. Pak, K. No, $\mathrm{AC}$ frequency characteristics of coplanar impedance sensors as design parameters, Lab Chip 5 (3) (2005) 270-279. doi:10.1039/B410325D.

[13] M. Shaker, L. Colella, F. Caselli, P. Bisegna, P. Renaud, An impedancebased flow micro-cytometer for single cell morphology discrimination, Lab Chip 14 (14) (2014) 2548-2555. doi:10.1039/c4lc00221k.

[14] C. Grenvall, C. Antfolk, C. Bisgaard, T. Laurell, Two-dimensional acoustic particle focusing enables sheathless chip Coulter counter with planar electrode configuration, Lab Chip 14 (24) (2014) 4629-4637. doi:10.1039/C4LC00982G.

[15] C. H. Clausen, G. E. Skands, C. V. Bertelsen, W. E. Svendsen, Coplanar electrode layout optimized for increased sensitivity for elec- 
trical impedance spectroscopy, Micromachines 6 (1) (2015) 110-120. doi:10.3390/mi6010110.

[16] V. Errico, A. De Ninno, F. Bertani, L. Businaro, P. Bisegna, F. Caselli, Mitigating positional dependence in coplanar electrode coulter-type microfluidic devices, Sens. Act. B Chem.doi:10.1016/j.snb.2017.03.035.

[17] T. Sun, N. G. Green, S. Gawad, H. Morgan, Analytical electric field and sensitivity analysis for two microfluidic impedance cytometer designs, IET Nanobiotechnol. 1 (5) (2007) 69-79. doi:10.1049/iet-nbt:20070019.

[18] J. Riordon, N. M.-Catafard, M. Godin, Using the fringing electric field in microfluidic volume sensors to enhance sensitivity and accuracy, Appl. Phys. Lett. 101 (15) (2012) 154105. doi:10.1063/1.4759033.

[19] A. De Ninno, V. Errico, F. Bertani, L. Businaro, P. Bisegna, F. Caselli, Coplanar electrode microfluidic chip enabling accurate sheathless impedance cytometry, Lab Chipdoi:10.1039/C6LC01516F.

[20] K. R. Foster, H. P. Schwan, Dielectric properties of tissues and biological materials: a critical review, CRC Crit. Rev. Biomed. Eng. 17 (1) (1989) 25-104.

[21] D. Di Carlo, Inertial microfluidics, Lab Chip 9 (21) (2009) 3038-3046. doi:10.1039/b912547g.

[22] M. Spiga, G. L. Morini, A symmetric solution for velocity profile in laminar flow through rectangular ducts, Int Commun Heat Mass 21 (4) (1994) 469-475. doi:10.1016/0735-1933(94)90046-9. 
[23] U. Hassan, R. Bashir, Coincidence detection of heterogeneous cell populations from whole blood with coplanar electrodes in a microfluidic impedance cytometer, Lab Chip 14 (22) (2014) 4370-4381. doi:10.1039/c4lc00879k.

[24] D. Spencer, F. Caselli, P. Bisegna, H. Morgan, High accuracy particle analysis using sheathless microfluidic impedance cytometry, Lab Chip 16 (13) (2016) 2467-2473. doi:10.1039/c6lc00339g.

[25] F. Caselli, P. Bisegna, A simple and robust event-detection algorithm for single-cell impedance cytometry, IEEE Trans Biomed Eng 63 (2) (2016) 415-422. doi:10.1109/TBME.2015.2462292.

[26] S. Gawad, L. Schild, P. Renaud, Micromachined impedance spectroscopy flow cytometer for cell analysis and particle sizing, Lab Chip 1 (1) (2001) 76-82. doi:10.1039/B103933B.

[27] D. A. Boy, F. Gibou, S. Pennathur, Simulation tools for lab on a chip research: advantages, challenges, and thoughts for the future, Lab Chip 8 (9) (2008) 1424-1431. doi:10.1039/B812596C.

[28] D. Erickson, Towards numerical prototyping of labs-on-chip: modeling for integrated microfluidic devices, Microfluid Nanofluid 1 (4) (2005) 301-318. doi:10.1007/s10404-005-0041-z.

[29] D. Das, K. Biswas, S. Das, A microfluidic device for continuous manipulation of biological cells using dielectrophoresis, Med Eng Phys 36 (6) (2013) 726-731. doi:10.1016/j.medengphy.2013.12.010. 
[30] P. Occhetta, M. Licini, A. Redaelli, M. Rasponi, Design of a microfluidic strategy for trapping and screening single cells, Med Eng Phys 38 (1) (2015) 33-40. doi:10.1016/j.medengphy.2015.10.009.

[31] F. Caselli, M. Shaker, L. Colella, P. Renaud, P. Bisegna, Modeling, simulation and performance evaluation of a novel microfluidic impedance cytometer for morphology-based cell discrimination, J. Microelectromech. Syst. 23 (4) (2014) 785-794. doi:10.1109/JMEMS.2014.2325979.

[32] Z. Zhu, X. Xu, L. Fang, D. Pan, Q. an Huang, Investigation of geometrydependent sensing characteristics of microfluidic electrical impedance spectroscopy through modeling and simulation, Sens. Act. B Chem. 235 (2016) 515-524. doi:10.1016/j.snb.2016.05.092.

[33] F. Caselli, P. Bisegna, F. Maceri, EIT-inspired microfluidic cytometer for single-cell dielectric spectroscopy, J. Microelectromech. Syst. 19 (5) (2010) 1029-1040. doi:10.1109/JMEMS.2010.2067204.

[34] D. Spencer, H. Morgan, Positional dependence of particles in microfludic impedance cytometry, Lab Chip 11 (7) (2011) 1234-1239. doi:10.1039/c1lc20016j. 